\title{
Wulff Shape of Strontium Titanate Nanocuboids
}

${\text { Lawrence C } \text { rosby }^{\mathrm{a}}{ }^{*} \text {, James Enterkin }}^{\mathrm{b}}$, Federico Rabuffetti ${ }^{\mathrm{c}}$, Ken Poeppelmeier ${ }^{\mathrm{b}}$ and Laurence Marks $^{\mathrm{a}}$

${ }^{\mathrm{a}}$ Department of $\mathrm{M}$ aterials $\mathrm{S}$ cience and $\mathrm{E}$ ngineering, $\mathrm{N}$ orthwestern $\mathrm{U}$ niversity, $\mathrm{E}$ vanston, I L 60208

${ }^{b}$ Department of Chemistry, Northwestern University, Evanston, IL 60208

${ }^{\mathrm{c}}$ Department of Chemistry, Wayne State University, Detroit, MI 48202

Keywords: Wulff Construction, transmission electron microscopy, nanocuboid, hydrothermal synthesis, strontium titanate, Kirkendall Effect

\section{Abstract}

Here we describe the Wulff shape of strontium titanate nanocuboids prepared by a hydrothermal method and a nnealed at high temperature. Transmission electron microscopy was us ed to measure the faceting ratios $d_{(110)}: d_{(100)}$ which are compared with surface energy ratios $\gamma_{(110)}: \gamma_{(100)}$ from first-principles c alculations. Internal vo ids a ttributed to the $\mathrm{K}$ irkendall e ffect w ere a lso measured and show agreement with the external faceting. Experiment and theory are shown to agree strongly within statistical and density functional theory error.

*Corresponding author: crosbyla@u.northwestern.edu 


\section{Introduction}

Oxide materials have been developed for a broad array of applications ranging from catalysis [1], to dielectrics [2], ferroelectrics [3], and transparent conductors [4, 5]. O ne material which has been studied in detail is $\mathrm{s}$ trontium ti tanate $\left(\mathrm{SrTiO}_{3}\right.$ or $\left.\mathrm{S} \mathrm{TO}\right)$ due to its prototypical cubic perovskite crystal structure [6] and its widespread us e as a substrate for growth of thin films. There ha ve be en s everal $\mathrm{w}$ orks publ ished $\mathrm{r}$ egarding $\mathrm{s}$ ynthesis of $\mathrm{S}$ TO i n va rious na noscale morphologies [7-9]. In s pite of the considerable vol ume of 1 iterature on the subject, there is limited understanding of the properties of S TO in nanoparticle form. It is a lso important to consider that surfaces are distinct from the bulk due to the loss of coordination going from an "infinite" periodic structure to an abrupt termination of the said periodicity [10]. Given that the surface-to-volume ratio increases as particle size decreases, the properties of STO nanoparticles could be quite different from bulk STO.

It is well-known $\mathrm{t}$ hat the nanoparticle s hape $\mathrm{i} \mathrm{s}$ t hermodynamically controlled by the thermodynamic Wulff construction [11]. This is the surface that minimizes the total surface free energy of a crystal, and is found by taking the inner envelope of tangents of the surface energy as a function of crystallographic orientation. As such, the coverage of different facets will be fixed for a particular material system in thermodynamic equilibrium [12].

In this note we r eport the $\mathrm{W}$ ulff s hape of S TO a nnealed in a ir us ing electron m icroscopy to measure both the external shape as well as that of internal Kirkendall voids.

\section{Methods}

Strontium titanate nanocuboids were prepared by hydrothermal synthesis as described elsewhere [13-16]. The samples were dispersed on SiN TEM grids and subsequently annealed at different temperatures $\left(700^{\circ} \mathrm{C}\right.$ to $950^{\circ} \mathrm{C}$ in steps of $\left.50^{\circ} \mathrm{C}\right)$ for various times in a fused silica tube within a tube furnace. A JEOL JEM-2100 FasTEM was used for TEM imaging and electron diffraction (TED) $\mathrm{m}$ easurements. The T ED m easurements $\mathrm{w}$ ere us ed to determine the crystallographic orientation and characterize defects in the nanocuboids. Diffraction patterns were captured using the CCD camera on the microscope and averaged over several exposures.

Calculations for the observed Wulff shape were carried out by measuring the distance from the center of se veral $n$ anocuboids to the r espective face ( either (100) or (110) in o ur c ase) a nd yielded the $d_{(110)}: d_{(100)}$ ratios which are proportional to the ratios of surface free energy per area. All na nocuboids a nalyzed in this fashion w ere i maged $\mathrm{n}$ ear $\mathrm{t}$ o the [001] zone a xis a nd the arithmetic mean of several measurements was calculated for each anneal temperature (see Table 1).

DFT calculations were performed with the all-electron augmented plane wave +1 ocal orb itals WIEN2K c ode [17]. The surf ace $\mathrm{i}$ n-plane 1 attice $\mathrm{p}$ arameters $\mathrm{w}$ ere se $\mathrm{t} t \mathrm{o}$ hose $\mathrm{f}$ or the corresponding DFT optimized bulk cell, with $\sim 1.6 \mathrm{~nm}$ of vacuum to avoid errors within the DFT calculations as well as in the STM simulations, the latter being done using the Tersoff-Hamann approximation [18]. Muffin-tin radii were s et to 1.55, 2.36 a nd 1.75 Bohrs for O, S r a nd Ti respectively, as well as a $\min (\mathrm{RMT}) * \mathrm{~K}_{\max }$ of 7.0 , with a $3 \times 3 \times 1$ Brillouin-zone reciprocal space sampling of the pr imitive uni $\mathrm{t}$ cell. $\mathrm{T}$ he electron density and atomic pos itions $\mathrm{w}$ ere 
simultaneously converged using a quasi-Newton algorithm [19]; the numerical convergence was better than $0.01 \mathrm{eV} / 1 \mathrm{x} 1$ surface cell. The PBEsol [20] generalized gradient approximation as well as the revTPSS method [21] was used, with 0.5 on-site exact-exchange the optimized number for several test $\mathrm{TiO}_{\mathrm{x}}$ molecules similar to earlier work [22]. The surface enthalpy per (1x1) surface unit cell $\left(E_{\text {surf }}\right)$ was calculated as: $E_{\text {surf }}=\left(E_{\text {slab }}-E_{S T O} * N_{S T O}-E_{T O} * N_{T O}\right) /\left(2 * N_{1 \times 1}\right)$, where $E_{\text {slab }}$ is the total enthalpy of the slab, $E_{\text {STO }}$ for one bulk $\mathrm{SrTiO}_{3}$ unit cell, $N_{\text {STO }}$ the number of bulk $\mathrm{SrTiO}_{3}$ unit cells, $E_{T O}$ bulk rutile $\mathrm{TiO}_{2}, N_{T O}$ the number of excess $\mathrm{TiO}_{2}$ units and $\left(N_{1 \times 1}\right)$ the number of $(1 \times 1)$ c ells. $C$ onsistency checks b etween the different $f$ unctionals indicated an error in the energies of approximately $0.1 \mathrm{eV} / 1 \mathrm{x} 1$ cell $\left(\sim 60 \mathrm{~mJ} / \mathrm{m}^{2}, 8 \mathrm{~kJ} / \mathrm{mole}\right)$.

As a cave at, DFT c alculations are substantially better for relative en ergies than abs olute ones. Common, $\mathrm{s}$ imple $\mathrm{f}$ unctionals ba dly ove restimate t he covalency, leading $\mathrm{t}$ ot $\mathrm{oo} \mathrm{m}$ uch hybridization of the oxygen $2 p$ and metal $d$ s tates. While it is common to use LDA+U methods to correct this, we prefer an on-site exact exchange method as this leads to an effective $U$ which varies as a function of metal co-ordination so is more appropriate. In addition to this, the use of a metaGGA leads to a m uch better treatment of the states at surfaces, and much better surfaces errors. However, there will still be systematic errors, for instance the non-bonded O-O repulsions are probably under-estimated.

\section{Results}

The $r$ esults of T EM i maging $r$ eveal a ge neral $c$ ubic morphology with the (100) facets dominating, but with additional significant coverage of (110) faces. The nanocuboids were single crystals as e videnced by the na nodiffraction measurements (see Figure 3). HREM demonstrate that faces which appear flat at low magnifications have many defects and step edges, which are a combination of the (100) and ( 110) f aces. There w ere al so de fects pr esent within the nanocuboids $w$ ith $t$ he $s$ ame $s$ hape a nd faceting as the ext erior surfaces. Thickness $m$ apping measurements show ed that these areas were thinner than the rest of the na nocuboids and thus they were identified as voids or cavities. Such voids have been observed in other work $([23,24])$, and in our case it is likely that they result from interdiffusion of the strontium precursor into an amorphous titanium complex [25] during the hydrothermal treatment.

A t able w ith measurements of $d_{(110)}: d_{(100)}$ ratios for va rious anne aling t emperatures appe ars below (Table 1). The a verage $d_{(110)}: d_{(100)}$ ratio calculated is 1.139 with a standard deviation of 0.055 . With a high degree of statistical confidence, increasing the annealing temperature had no effect on the faceting. However, it did reduce the corner rounding and faceting by reducing the number of surface $s$ teps. S amples a nnealed at $700^{\circ} \mathrm{C}$ e xhibited di stinct (110) faceting a t the corners (see Figure 3a). Increasing the temperature further resulted in smoother (100) and (110) faces (e.g. Figure $3 \mathrm{~b}$ ) until the po int that s urface roughening oc curred at $1100^{\circ} \mathrm{C}$. The voi ds observed in the as-prepared samples remained after the annealing process. The voids measured were found to exhibit the same faceting as the external surfaces, and the size remained the same after annealing (see Figure 3).

A car eful analysis of a nu mber of particles us ing images near the [111] and [110] z one axes indicated that there was no e vidence of a ny (111) facets, and ve ry little of a ny o ther type of 
exposed surface excepting some rounding of what would otherwise be sharp corners (see Figures 3 and 4).

\section{Discussion}

The $\mathrm{f}$ act $\mathrm{t}$ hat $\mathrm{t}$ he na nocuboids largely $\mathrm{m}$ aintained $\mathrm{t}$ heir $\mathrm{s}$ hape be fore a nd a fter annealing a $\mathrm{s}$ evidenced by the $d_{(110)}: d_{(100)}$ ratios remaining statistically unchanged indicates that the shape we have observed is the thermodynamic limit and is representative of the Wulff construction. Other shapes have been observed such as the cubic morphology in which the (100) surfaces dominate, but the synthesis surfactant is likely a limiting reagent for crystallization on the (110) surfaces, and as suc $h$ a predom inantly kinetic ef fect. In our case, the annealing process p rovides an explanation for the stabilization of the (110) facets both on the surfaces of the nanocuboids and internally in the voids.

The c omparison of the e xperimental results $\mathrm{w}$ ith DFT c alculations is ve ry informative, remembering that the latter are better at yielding relative energies than absolute values. A table of cal culated surface ene rgy ratios for va rious s urface s tructures on (100) a nd (110) s urfaces based upon the D FT calculations i s g iven in Figure 5. A m ore com plete c omparison t o experiment is to compare the ratio $\gamma_{(110)}: \gamma_{(100)}$, u sing a $\mathrm{n}$ e rror of $0.13 \mathrm{f}$ or the DFT c alculations based upon a $\mathrm{n}$ unc ertainty of $0.1 \mathrm{eV} / 1 \mathrm{x} 1$ unit c ell. Based upon $\mathrm{r}$ ecent work [26] the samples should be near the composition of the $\sqrt{ } 13 x \sqrt{ } 13$ r econstruction [27], perhaps co-existing with some SrO 1x1 termination. For the (110) surface energy we used a linear combination of the 1x1 oxygen terminated surface and the $3 \times 1$ surface [28].

The agreem ent is reasonable, although the ratio expected from the DFT cal culations is 1 arger than one would expect for a $\sqrt{13} x \sqrt{ } 13$ reconstruction.

\section{Acknowledgements}

We a cknowledge $\mathrm{f}$ unding f rom $\mathrm{N}$ orthwestern $\mathrm{U}$ niversity $\mathrm{I}$ nstitute $\mathrm{f}$ or $\mathrm{C}$ atalysis i $\mathrm{n} \mathrm{E}$ nergy Processes (ICEP) on gr ant num ber D OE D E-FG02-03-ER15457 (JAE, FR) a nd the N ational Science F oundation on G rant N umber DMR-1206320 (LC and LDM). LC a lso a cknowledges support from a National Science Foundation Graduate Fellowship. 

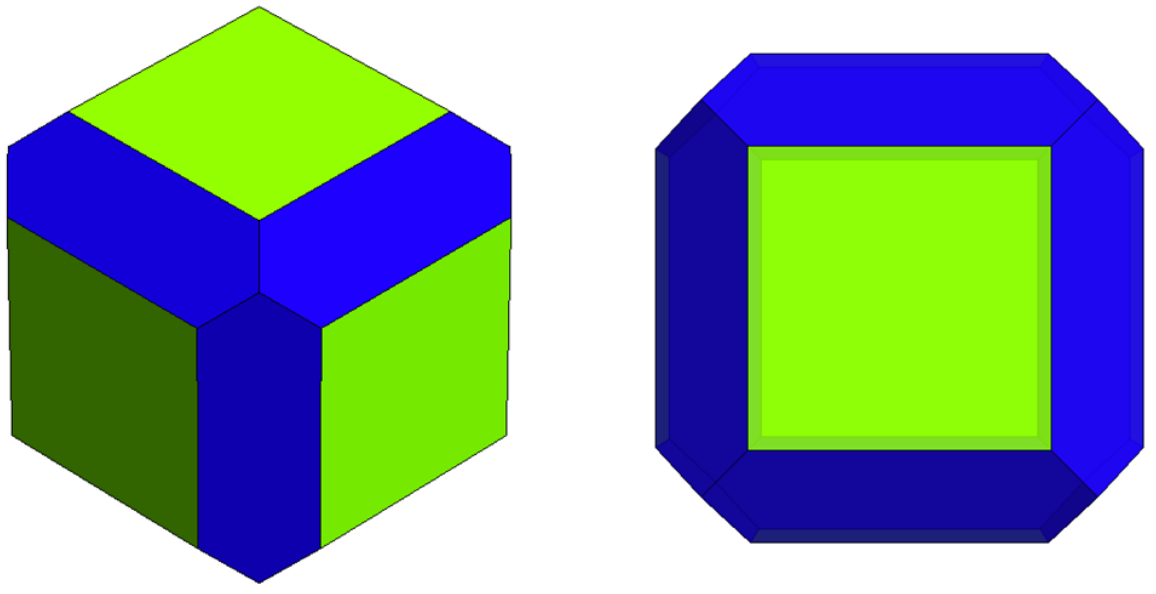

Figure 1: Observed octadecahedral shape of $\mathrm{SrTiO}_{3}$ nanoparticles. Note that the coverage of the six $\{100\}$ facets (green) dominates and thus they are described as nanocuboids. The shape was calculated using the WulffMaker Mathematica code [29] for the $d_{(110)}: d_{(100)}$ ratio of 1.139.

Table 1: Measured $d_{(110)}: d_{(100)}$ ratios for nanocuboids following various annealing conditions.

\begin{tabular}{ccccc}
\hline $\begin{array}{c}\text { Annealing } \\
\text { Conditions }\end{array}$ & $\begin{array}{c}\text { \# Cuboids } \\
\text { Measured }\end{array}$ & $\begin{array}{c}\text { Mean } \\
d_{(110)}: d_{(100)} \\
\text { Ratio }\end{array}$ & $\begin{array}{c}\text { Standard } \\
\text { Deviation }\end{array}$ & Margin of Error \\
\hline $700^{\circ} \mathrm{C}, 5 \mathrm{~h}, \mathrm{Air}$ & 2 & 1.173 & 0.032 & 0.286 \\
$750^{\circ} \mathrm{C}, 10 \mathrm{~h}, \mathrm{Air}$ & 3 & 1.130 & 0.039 & 0.096 \\
$800^{\circ} \mathrm{C}, 5 \mathrm{~h}, \mathrm{Air}$ & 3 & 1.198 & 0.038 & 0.342 \\
$950^{\circ} \mathrm{C}, 2 \mathrm{~h}, \mathrm{O} 2$ & 8 & 1.140 & 0.026 & 0.052 \\
$950^{\circ} \mathrm{C}, 5 \mathrm{~h}, \mathrm{O} 2$ & 6 & 1.160 & 0.050 & 0.022 \\
$950^{\circ} \mathrm{C}, 20 \mathrm{~h}, \mathrm{O} 2$ & 9 & 1.107 & 0.086 & 0.066 \\
\hline Total & 29 & 1.139 & 0.055 & 0.045
\end{tabular}
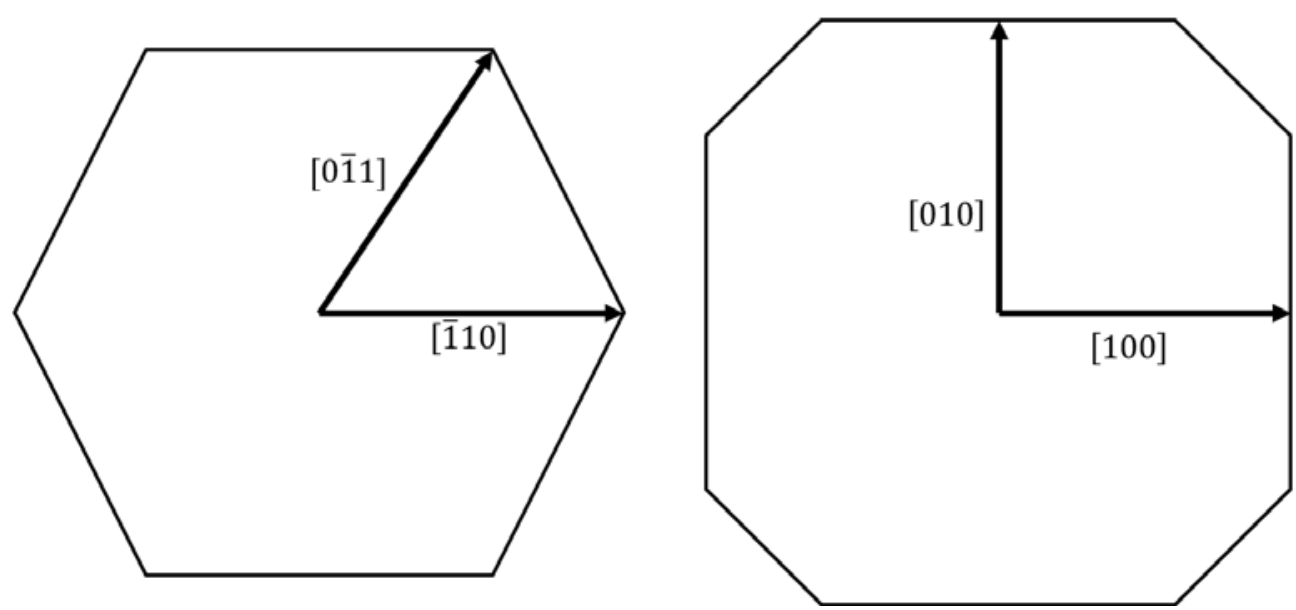

Figure 2: Projection of nanocuboid along left [111] zone axis and right [001] zone axis 

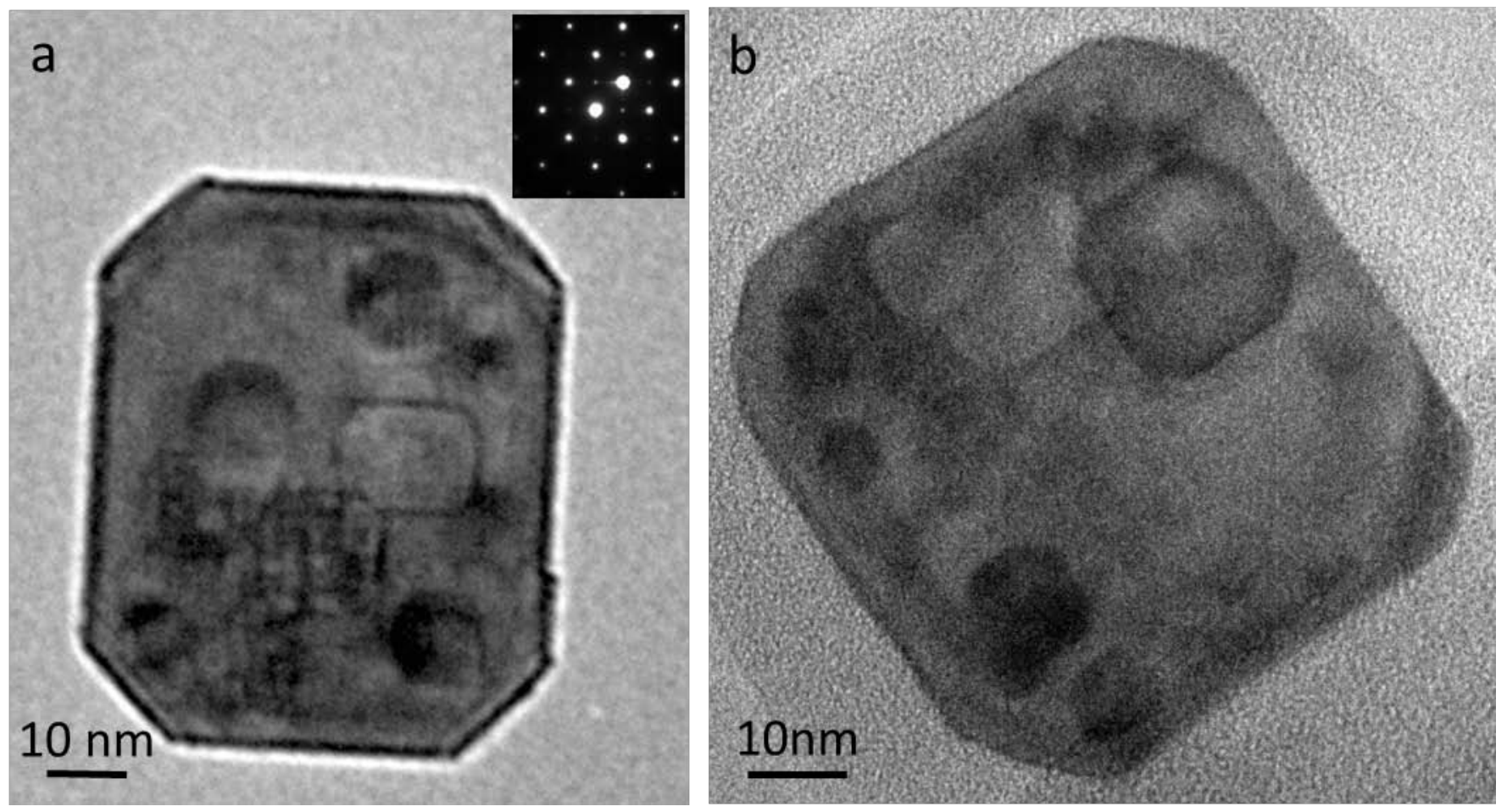

Figure 3: TEM images of annealed nanocuboids a long [001] zone axis de monstrating distinct (100) and (110) faceting that is characteristic of the Wulff shape, in a) annealed at $700^{\circ} \mathrm{C}$ and b) $900^{\circ} \mathrm{C}$. 

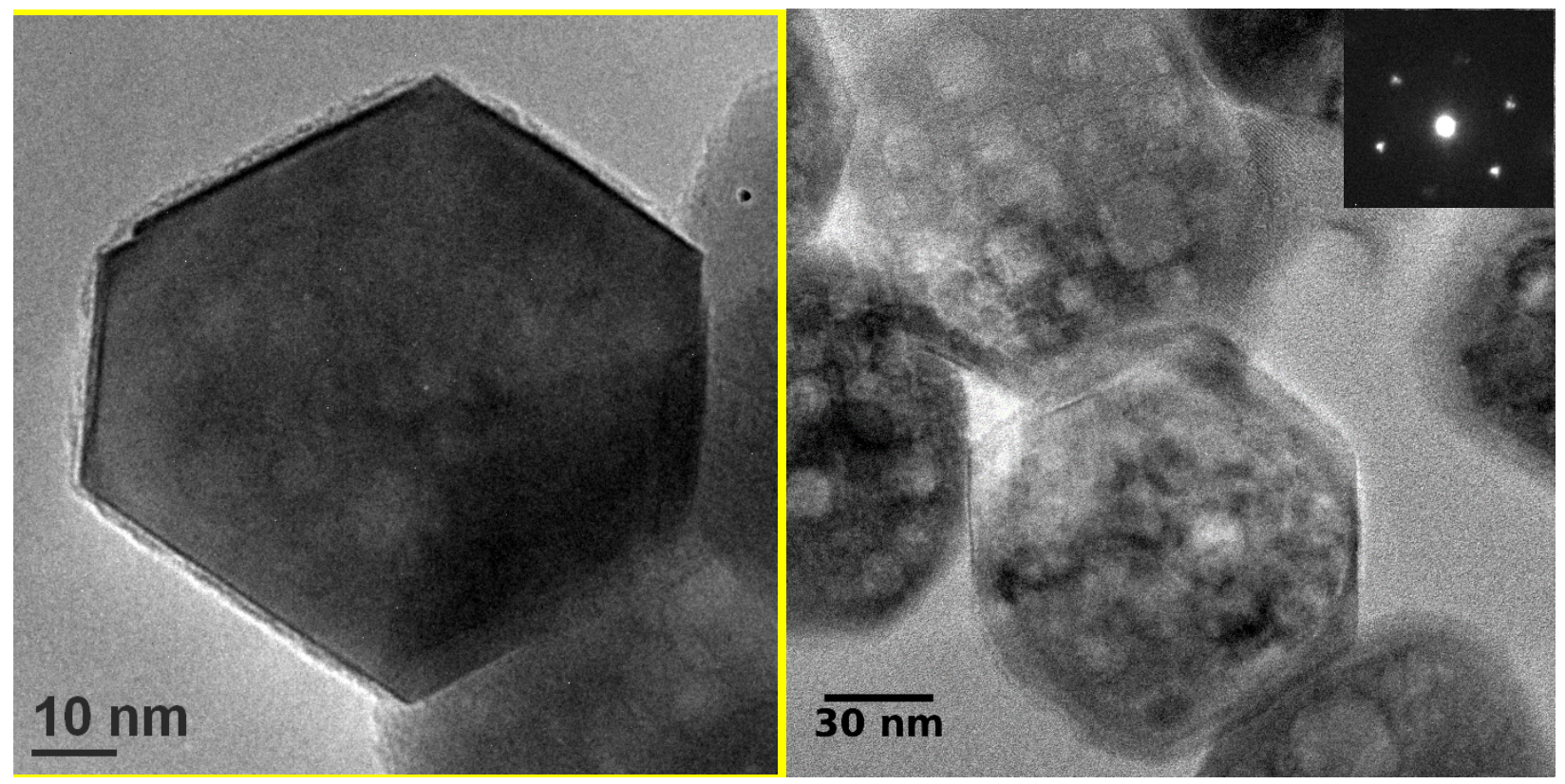

Figure 4: TEM Images of several nanocuboids (also annealed) near [111] zone axis. Note that in both i mages, the projected shape is hexagonal, indicating that no substantial (111) faceting is present.

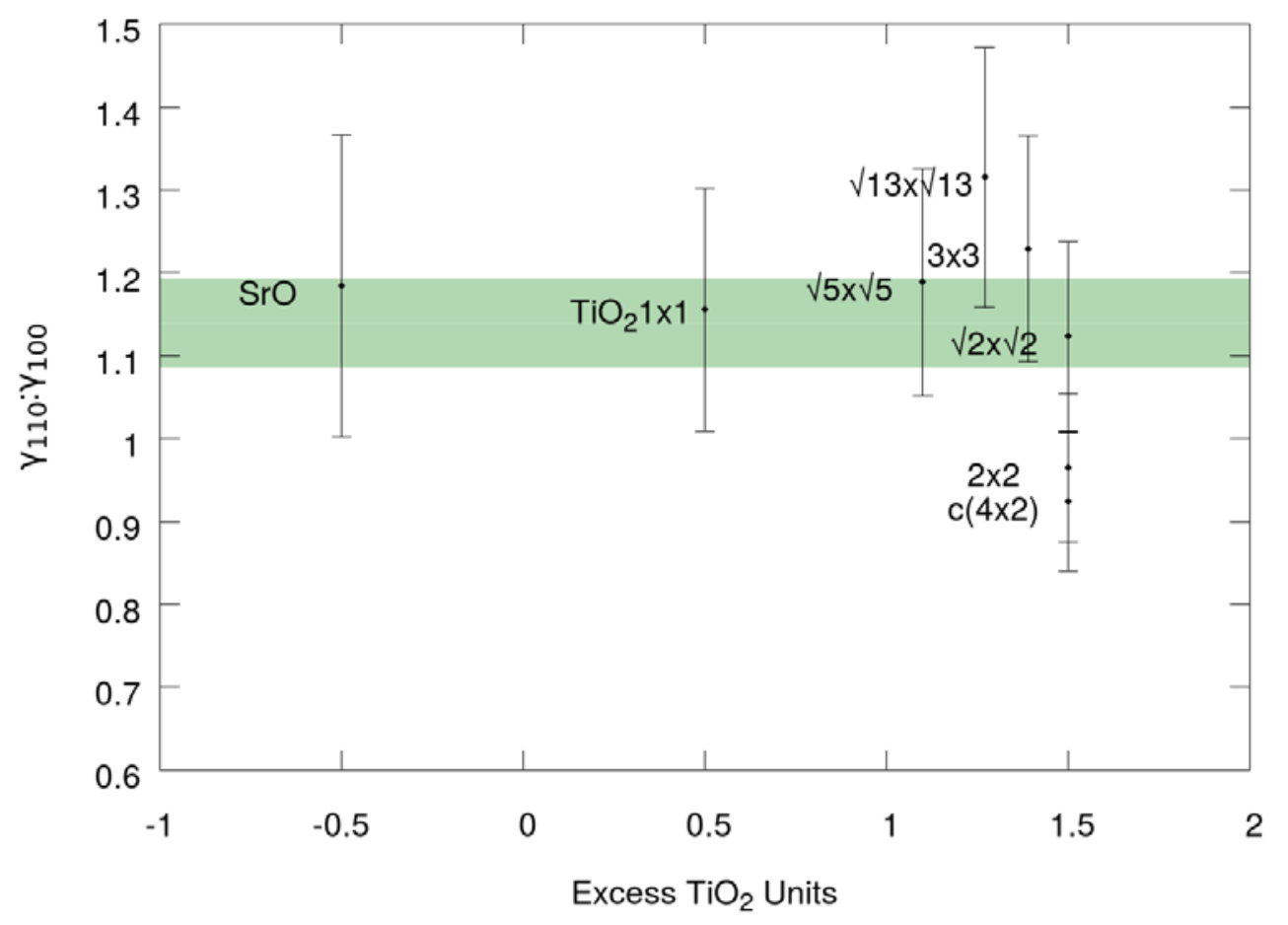

Figure 5: $\gamma_{(110)}: \gamma_{(100)}$ ratios $\mathrm{f}$ rom $\mathrm{f}$ irst-principles $\mathrm{c}$ alculations $\mathrm{c}$ ompared $\mathrm{w}$ ithin o ne $\mathrm{s}$ tandard deviation of the mean observed $d_{(110)}: d_{(100)}$ ratio of 1.139 . 


\section{References}

[1] A.R.J.H. Voorhoeve, D.W. Johnson, J.P. Remeika, P.K. Gallagher, Perovskite Oxides : Materials Science in Catalysis, Science, 195 (1977) 827-833.

[2] G. Arlt, D. Hennings, G.d. With, Dielectric properties of fine-grained barium titanate ceramics, in: Journal of Applied Physics, 1985, pp. 1691-1625.

[3] K. Kiss, J. Magder, M.S. Vukasovich, R.J. Lockhart, Ferroelectrics of Ultrafine Particle Size: I, Synthesis of Titanate Powders of Ultrafine Particle Size, Journal of the American Ceramic Society, 49 (1966) 291-295.

[4] L. Goris, R. Noriega, M. Donovan, J. Jokisaari, G. Kusinski, a. Salleo, Intrinsic and Doped Zinc Oxide Nanowires for Transparent Electrode Fabrication via Low-Temperature Solution Synthesis, Journal of Electronic Materials, 38 (2008) 586-595.

[5] A. Klein, C. Körber, A. Wachau, F. Säuberlich, Y. Gassenbauer, S.P. Harvey, D.E. Proffit, T.O. Mason, Transparent Conducting Oxides for Photovoltaics: Manipulation of Fermi Level, Work Function and Energy Band Alignment, Materials, 3 (2010) 4892--4914.

[6] A. Bhalla, R. Guo, R. Roy, The perovskite structure-a review of its role in ceramic science and technology, Material Research Innovations, (2000) 3-26.

[7] K. Fujinami, K. Katagiri, J. Kamiya, T. Hamanaka, K. Koumoto, Sub-10 nm strontium titanate nanocubes highly dispersed in non-polar organic solvents., Nanoscale, 2 (2010) 2080-2083.

[8] U.a. Joshi, J.S. Lee, Template-free hydrothermal synthesis of single-crystalline barium titanate and strontium titanate nanowires, Small (Weinheim an der Bergstrasse, Germany), 1 (2005) 1172-1176.

[9] J. Urban, W. Yun, Q. Gu, H. Park, Synthesis of single-crystalline perovskite nanorods composed of barium titanate and strontium titanate, Journal of the American ..., 124 (2002) 1186-1187.

[10] G.A. Somorjai, Principles of Surface Chemistry, (1972).

[11] G. Wulff, On the question of speed of growth and dissolution of crystal surfaces, Zeitschrift fur Krystallographie und Mineralogie, 34 (1901) 449-530.

[12] S. Miracle-Sole, Wulff shape of equilibrium crystals, arXiv preprint arXiv:1307.5180, (2013) 1-15.

[13] F.A. Rabuffetti, H.S. Kim, J.A. Enterkin, Y.M. Wang, C.H. Lanier, L.D. Marks, K.R. Poeppelmeier, P.C. Stair, Synthesis-dependent first-order Raman scattering in SrTi03 nanocubes at room temperature, Chemistry of Materials, 20 (2008) 5628-5635.

[14] S.T. Christensen, J.W. Elam, F.A. Rabuffetti, Q. Ma, S.J. Weigand, B. Lee, S. Seifert, P.C. Stair, K.R. Poeppelmeier, M.C. Hersam, M.J. Bedzyk, Controlled Growth of Platinum Nanoparticles on Strontium Titanate Nanocubes by Atomic Layer Deposition, Small, 5 (2009) 750-757.

[15] J.A.P. Enterkin, Kenneth R and Marks, Laurence D Oriented Catalytic Platinum Nanoparticles on High Surface Area Strontium Titanate Nanocuboid Surfaces, Nanoletters, 11 (2011) 993-997. 
[16] F.A. Rabuffetti, P.C. Stair, K.R. Poeppelmeier, Synthesis-Dependent Surface Acidity and Structure of SrTiO3 Nanoparticles, Journal of Physical Chemistry C, 114 (2010) 1105611067.

[17] P. Blaha, K. Schwarz, G. Madsen, D. Kvasnicka, J. Luitz, Wien2k, an augmented plane wave + local orbitals program for calculating crystal properties in, Techn. Universität Wien, Austria, 2001.

[18] J. Tersoff, D.R. Hamann, Theory and application for the scanning tunneling microscope, Phys Rev Lett, 50 (1983) 1998-2001.

[19] L.D. Marks, Fixed-point optimization of atoms and density in DFT, J Chem Theory Comput, 9 (2013) 2786-2800.

[20] J.P. Perdew, A. Ruzsinszky, G.I. Csonka, O.A. Vydrov, G.E. Scuseria, L.A. Constantin, X.L. Zhou, K. Burke, Restoring the density-gradient expansion for exchange in solids and surfaces, Phys Rev Lett, 100 (2008).

[21] V.N. Staroverov, G.E. Scuseria, J. Tao, J.P. Perdew, Comparative assessment of a new nonempirical density functional: Molecules and hydrogen-bonded complexes, J Chem Phys, 119 (2003) 12129-12137.

[22] D.M. Kienzle, A.E. Becerra-Toledo, L.D. Marks, Vacant-site octahedral tilings on $\mathrm{SrTiO}_{3}$ (001), the $(\sqrt{13} \times \sqrt{13}) \mathrm{R} 33.7$ degrees surface, and related structures, Phys Rev Lett, 106 (2011).

[23] A.D. Smigelskas, E.O. Kirkendall, Zinc Diffusion in Alpha Brass, AIME Transactions, 171 (1947) 130-142.

[24] J. Chen, K. Huang, S. Liu, Hydrothermal preparation of octadecahedron Fe304 thin film for use in an electrochemical supercapacitor, Electrochimica Acta, 55 (2009) 1-5.

[25] Y. Zhu, L. Zhang, C. Gao, L. Cao, The synthesis of nanosized TiO2 powder using a sol-gel method with TiCl4 as a precursor, Journal of materials science, 5 (2000) 4049-4054.

[26] Y. Lin, J. Wen, L. Hu, R.M. Kennedy, P.C. Stair, K.R. Poeppelmeier, L.D. Marks, Synthesisdependent atomic surfaces structures on oxide nanoparticles, Phys Rev Lett, 111 (2013) 156101.

[27] D.M. Kienzle, A.E. Becerra-Toledo, L.D. Marks, Vacant-Site Octahedral Tilings on SrTi03 (001), the (root 13 x root 13)R33.7 degrees Surface, and Related Structures, Phys Rev Lett, $106(2011) 176102$.

[28] J.A. Enterkin, A.K. Subramanian, B.C. Russell, M.R. Castell, K.R. Poeppelmeier, L.D. Marks, A homologous series of structures on the surface of $\mathrm{SrTiO}_{3}(110)$, Nature Materials, 9 (2010) 245-248.

[29] R.V. Zucker, D. Chatain, U. Dahmen, S. Hagège, W.C. Carter, New software tools for the calculation and display of isolated and attached interfacial-energy minimizing particle shapes, Journal of Materials Science, 47 (2012) 8290-8302. 